\title{
In-flight Characterization and Calibration of the Juno-ultraviolet Spectrograph (Juno-UVS)
}

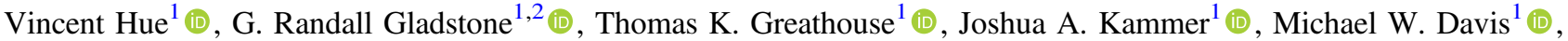

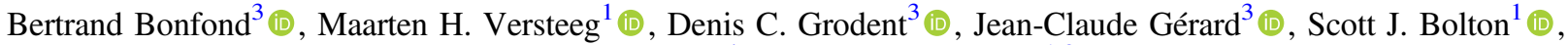 \\ Steven M. Levin ${ }^{4}$ (D), and Ben D. Byron ${ }^{1,2}$ \\ ${ }^{1}$ Southwest Research Institute, San Antonio, Texas, USA; vhue@swri.edu \\ ${ }^{2}$ University of Texas at San Antonio, San Antonio, Texas, USA \\ ${ }^{3}$ STAR Institute, LPAP, Université de Liège, Liège, Belgium \\ ${ }^{4}$ Jet Propulsion Laboratory, Pasadena, California, USA \\ Received 2018 October 16; revised 2018 December 28; accepted 2018 December 28; published 2019 February 1
}

\begin{abstract}
Juno is the first polar orbiter around Jupiter. Juno possesses a suite of instruments designed to measure the electron and ion populations in the Jupiter magnetosphere, leading to the powerful Jovian aurorae. The Ultraviolet Spectrograph onboard Juno (Juno-UVS) is a photon-counting imaging spectrograph $(68-210 \mathrm{~nm})$, designed to observe and characterize Jupiter's far-ultraviolet aurorae. The instrument borrows heavily from previous Alice and UVS instruments led by Southwest Research Institute (New Horizons and Rosetta Alices, LRO-LAMP), with several major improvements. The pointing flexibility offered by the UVS scan mirror combined with Juno's spin allows UVS access to half of the sky at any given moment. This paper describes how we leverage this extensive database to track the evolution of Juno-UVS calibration with time throughout the mission. UVS observes $7.2^{\circ} \times 360^{\circ}$-long swaths of the sky for each rotation of the spacecraft (nominally $2 \mathrm{rpm}$ ). This paper describes how the very substantial amount of stellar spectra has been used to monitor the health of the instrument over the mission. As of PJ14 (2018 July 16), more than 8700 spectra of O, A, and B stars have been extracted in the $V$-magnitude range of $\sim 0-7$, and more than $99 \%$ of the sky was mapped. Selected stars among this list were used to calibrate the UVS bandpass, using observations from the International Ultraviolet Explorer and the Hubble Space Telescope. The retrieved effective area of the instrument is $0.30 \pm 0.03 \mathrm{~cm}^{2}$ at $125 \mathrm{~nm}, 0.15 \pm 0.02 \mathrm{~cm}^{2}$ at $140 \mathrm{~nm}$, and $0.05 \pm 0.01 \mathrm{~cm}^{2}$ at $160 \mathrm{~nm}$.
\end{abstract}

Key words: instrumentation: spectrographs - methods: data analysis - stars: general - techniques: imaging spectroscopy - ultraviolet: stars

\section{Introduction}

Juno is a NASA New Frontiers mission launched on 2011 August 5 from Cape Canaveral Air Force Station on an United Launch Alliance Atlas V551 rocket (Bolton et al. 2017b). Juno was designed to study Jupiter, from its outer magnetosphere all the way down through its atmosphere and into the deep interior. To that purpose, it carries a suite of eight science instruments plus an outreach camera. After cruising for $5 \mathrm{yr}$, the Jupiter orbital insertion (JOI) was performed on 2016 July 4, placing Juno into a 53 day polar orbit. Juno's orbit is highly eccentric $(e=0.98)$, with perijoves at about $1.05 R_{J}$ from Jupiter's center, where $R_{J}$ stands for Jupiter's radius, and apojove at about 113 Jovian radii, or $R_{J}\left(1 R_{J}=71,492 \mathrm{~km}\right)$. Furthermore, Juno is a solar-powered, spin-stabilized spacecraft, nominally rotating at $2 \mathrm{rpm}$. The first science perijove (PJ) was on 2016 August 27, leading to a first glimpse of Jupiter's complexity (Bolton et al. 2017a; Connerney et al. 2017). More specifically, it showed how the remote sensing instruments greatly aid the interpretation of particle measurements (Mauk et al. 2017), and how Juno's vantage point in the Jovian system provides an unprecedented opportunity to study its auroral emissions (Bonfond et al. 2017; Gladstone et al. 2017b). The suite of remote sensing science instruments on Juno, built to provide context for the in situ instruments, are the Jupiter Infrared Auroral Mapper (JIRAM; Adriani et al. 2017) and the Ultraviolet Spectrograph (UVS; Gladstone et al. 2017a).
The focus of this paper is the characterization and in-flight calibration of the UVS instrument. UVS is a photon-counting imaging spectrograph with a bandpass spanning $68-210 \mathrm{~nm}$, designed to target the most prominent features of the Jovian far-ultraviolet (FUV) aurora (see review from Grodent 2015). These spectral features are created by energetic particles colliding with Jupiter's upper atmosphere, exciting $\mathrm{H}$ and $\mathrm{H}_{2}$, which then radiatively relax through emissions of the Lyman series of $\mathrm{H}$ (notably, $\operatorname{Ly} \alpha$ ), as well as the Lyman, Werner, and Rydberg band systems of $\mathrm{H}_{2}$. Juno-UVS operates in a scanning mode, constrained by Juno's spin period of $30 \mathrm{~s}$. The nominal field of view of UVS is a $7.2^{\circ}$-long slit oriented along Juno's spin axis and centered on Juno's spin plane. Thus, in a given spin of the spacecraft, UVS maps out a $360^{\circ} \times 7.2^{\circ}$ swath on the sky.

Juno-UVS is a heritage design based on three previous UVinstruments produced at the Southwest Research Institute (SwRI). These instruments are: (i) the Alice instrument on the Rosetta mission (Stern et al. 2007), which orbited comet 67P/ Churyumov Gerasimenko from 2014 August to 2016 September; (ii) the Alice instrument on the New Horizons mission (Stern et al. 2008), which flew by Pluto in 2015 July; and (iii) the Lyman Alpha Mapping Project (LAMP) on the Lunar Reconnaissance Orbiter (LRO) mission (Gladstone et al. 2010), continuously mapping the moon since 2009 September. The main differences between Juno-UVS and its predecessors are: (i) the addition of a scan mirror, allowing UVS to shift the instrument field of view by up to $\pm 30^{\circ}$ above or below the 
spacecraft spin plane; (ii) extensive shielding to protect the electronics and detector from Jupiter's high radiation environment; and (iii) a cross-delay microchannel plate (MCP) detector with an improved readout scheme $(2048 \times 256)$, providing higher spatial and spectral resolution, as well as higher input count rates, than heritage instruments.

One of the main challenges to fly and operate an MCP detector in a high radiation environment (such as Jupiter's inner magnetosphere) is the high background count rate triggered by penetrating radiation. MCP detectors are known to be sensitive to high-energy electrons and ions, as well as gamma-rays. Several laboratory measurements have been performed using a monoenergetic electron beam to quantify the detection efficiency of the Juno-UVS flight spare detector to electrons and gamma-rays (Davis et al. 2016). The measured efficiency of the UVS-like MCP was found to be $\sim 30 \%$ for electrons, regardless of their input energy in the $\sim 0.65-1.70 \mathrm{MeV}$ range. The measured MCP efficiency to gamma-rays was found to be $\sim 2.4 \%$ for gammas in the $\sim 0.03-0.4 \mathrm{MeV}$ range.

To successfully operate in Jupiter's high radiation environment, and to mitigate the high counting rates caused by these radiations, two main observational strategies were adopted and are extensively described in Kammer et al. (2018). Even with these strategies, the radiation background Juno-UVS experiences over a single PJ observation data set, which nominally runs $\pm 5 \mathrm{hr}$ around PJ, is quite substantial. With such high count rates, first the UVS detector gain, and then the detector sensitivity, can degrade substantially over time, so that frequent stellar calibrations are important.

The goal of this paper is to describe how the in-flight health of UVS is assessed on a regular basis, and how regular in-flight calibrations are performed. In the first place, we will first provide a description of the UVS instrument as well as how it is operated over a typical Juno orbit. We will present how the characterization of the UVS instrument is performed from launch to PJ14 (2018 July 16), and then we will present the method developed to perform the in-flight calibration of UVS. Finally, the calibration of the instrument is applied and presented here using this method.

\section{Instrument Description and Operation}

\subsection{Instrument Description}

Juno-UVS is composed of two separate assemblies. The instrument sensor housing, which holds the telescope, detector, and detector electronics is located on the spacecraft's deck, and is looking radially outward from Juno's spin axis (Bolton et al. 2017b). The command and data handling electronics (C\&DH) and power supplies are located in an electronics housing within the spacecraft vault to protect them from radiation. The two parts are linked by approximately $4 \mathrm{~m}$ of cable.

An overview of the telescope assembly and detector is provided in Figure 1 (left) and shows the trajectory followed by photons in color, with a different color after each reflection on an instrument optical surface. The photons entering the telescope first hit the scan mirror, which redirect them through a $4 \times 4 \mathrm{~cm}^{2}$ aperture toward an off-axis parabolic mirror (OAP). The OAP focuses the photons on a dog-bone-shaped slit, represented in Figure 1 (right). The dimensions of the slit are $2.55 \times 0.2$ for each of the wide outer sections, and $2^{\circ} \times 00^{\circ} .025$ for the narrow slit of the central section. The photons then hit the toroidal diffraction grating, mounted in a Rowland circle configuration with the slit and the microchannel plate (MCP) detector. This light is then diffracted and focused onto the detector. The aluminum scan mirror, OAP, and diffraction grating are coated with an aluminum and magnesium fluoride overcoat $\left(\mathrm{Al} / \mathrm{MgF}_{2}\right)$ to optimize reflectivity in the UVS spectral bandpass (e.g., Gladstone et al. 2017a, for a more detailed discussion).

The detector consists of three MCPs in a Z-stack configuration that are read out by a cross-delay line (XDL) anode. The Z-stack is cylindrically curved in order to match the $150 \mathrm{~mm}$ Rowland circle to optimize the spectral focus. The input surface of the Z-stack is coated with an opaque, solar-blind, UV-sensitive, Cesium Iodide (CsI) photocathode, providing good quantum detection efficiency over the UVS spectral bandpass (Siegmund 1999). The choice of that instrument design was made in order to achieve UVS science goals, i.e., observing and characterizing Jupiters FUV auroral emissions, while taking advantage of the heritage from previous successful instruments. The detectorpixel readout format is $2048 \times 256$ (spectral $x$ spatial) over the $4 \times 3 \mathrm{~cm}^{2}$ surface, and with an active detection array of $3.5 \times$ $1.8 \mathrm{~cm}^{2}$. Each pixel is $25 \mu \mathrm{m}$ and $89 \mu \mathrm{m}$ in the $X$ - and $Y$ - directions, respectively. This corresponds to $0.1020 \mathrm{~nm} \pm$ $0.0004 \mathrm{~nm}$ in the spectral direction $(X)$ and $0.041^{\circ} \pm 0.001^{\circ}$ in the spatial $(Y)$ direction. The detector electronics amplify the signal coming from the $\mathrm{MCP}$, and convert the detected output pulses to locate the $X$ (spectral) and $Y$ (spatial) coordinates of the detected events. Only those analog pulses output from the MCP that have amplitudes above a set discriminator level are processed and converted to pixel address locations (Stern et al. 2008; Gladstone et al. 2017a). In order to monitor the health of the instrument, i.e., the output gain of the detector at a given high-voltage (HV) level, the pulse heights are recorded for each event. The pulse height distribution (PHD) is produced by histogramming the pulse heights. The shape of a PHD can help to identify the sources of detected counts, UV photons, electrons, ions, or gamma-ray photons (Stern et al. 2007).

The C\&DH controls the high-voltage power supply (HVPS) level (and by default the detector gain), the detector heater, and actuator activation mechanisms, as well as the scan mirror mechanisms. Among other things, it also monitors the detector count rate, voltage, and strip current so that it can protect the instrument against anomalous conditions.

The spatial point-spread function (PSF) was measured during the high-voltage commissioning (HVC) and the periodic maintenance 1 (PM1). The description of the operations performed during these sequences were described in an earlier paper (Greathouse et al. 2013). The PSF is expected to vary along the slit because the spatial focus varies depending on the slit position. Previous work from Greathouse et al. (2013) showed that the PSF along the slit, i.e., parallel to the slit direction, varies almost linearly from $\sim 0.15^{\circ}$ up to $\sim 0.29^{\circ}$. The PSF across the slit, i.e., in the spin direction, is best in the narrow part of the slit, where it is $\sim 0.19^{\circ}$, and is $\sim 0.25^{\circ}$ over the wide slits. The spectral resolution of UVS is also slitdependent. It is best in the narrow slit $(\sim 1.3 \mathrm{~nm})$, and worst at the outermost ends of the wide slits $(\sim 3 \mathrm{~nm})$. The main characteristics and performances of the Juno-UVS instrument are summarized in Table 1. 

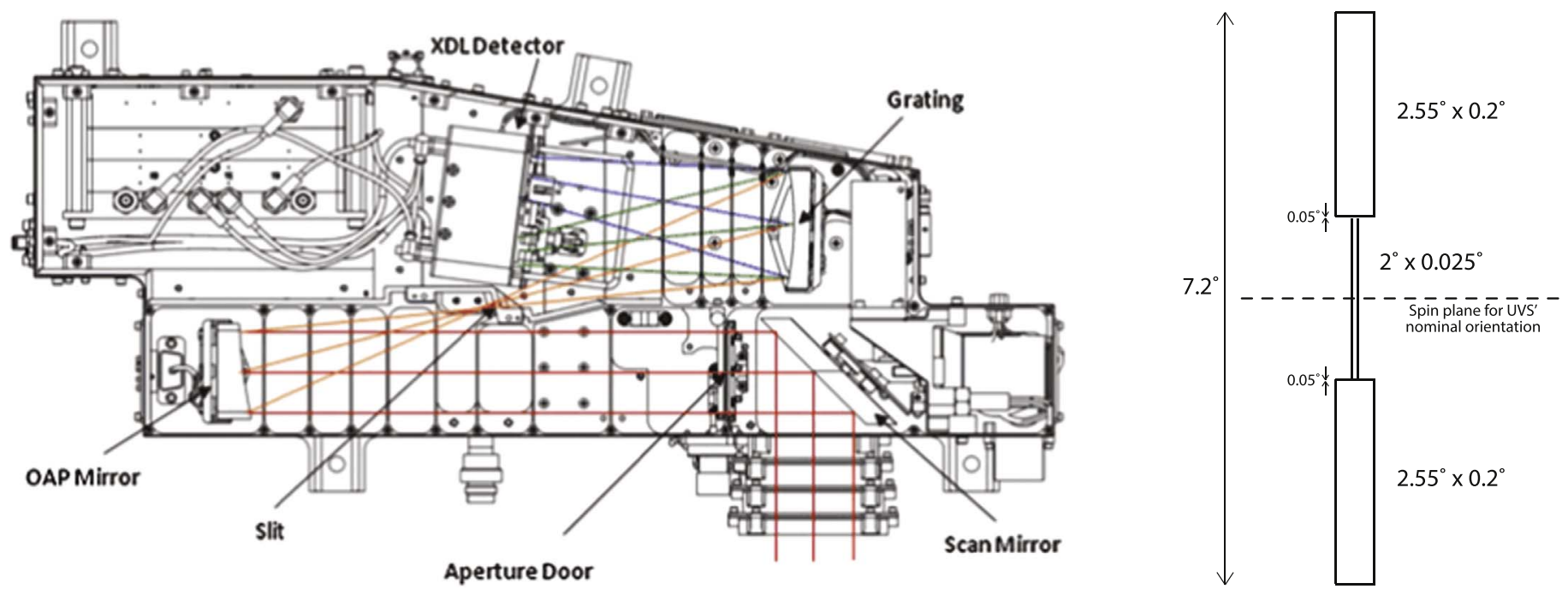

Figure 1. Left: schematic of the Juno-UVS sensor. Light is first reflected by the scan mirror. The primary (off-axis parabolic, OAP) mirror then focuses the light onto the slit and through the grating, which disperses the light onto the cross-delay microchannel plate (XDL detector) for imaging. Right: schematic of the dog-boneshaped slit, composed of two wide slits on either side of a much narrower slit.

Table 1

Overview on the Instrument Characteristics and Performances

\begin{tabular}{ll}
\hline \hline Spectral Range & $68-210 \mathrm{~nm}$ \\
\hline $\begin{array}{l}\text { Point source FWHM } \\
\text { along the slit }\end{array}$ & $\sim 0.15^{\circ}-0.29^{\circ}$ along the slit \\
$\begin{array}{l}\text { Point source FWHM } \\
\text { across the slit }\end{array}$ & $0.25^{\circ}$ (wide slits) $0.19^{\circ}$ (narrow slit) \\
$\begin{array}{l}\text { (i.e., in the spin } \\
\text { direction) }\end{array}$ & \\
\hline
\end{tabular}

\begin{tabular}{|c|c|}
\hline Spectral resolution & $\begin{array}{l}\sim 1.9-3.0 \mathrm{~nm} \text { (wide slits) } \\
1.3 \mathrm{~nm} \text { (narrow slit) }\end{array}$ \\
\hline Field of view & $\begin{array}{l}0.2^{\circ} \times 2.55^{\circ}+0.025^{\circ} \times 2^{\circ}+0.2^{\circ} \times 2.55^{\circ} \rightarrow \\
\quad \text { dog-bone-shaped }\end{array}$ \\
\hline Field of regards & $\begin{array}{l}360^{\circ} \times 60^{\circ} \\
\left(2 \mathrm{rpm} \text { and scan mirror allows } \pm 30^{\circ} \text { from spin plane }\right. \\
\quad \rightarrow \text { half the sky is accessible })\end{array}$ \\
\hline $\begin{array}{l}\text { Spectral }(X) \text { plate scale } \\
\text { Spatial }(Y) \text { plate scale }\end{array}$ & $\begin{array}{l}0.1020 \mathrm{~nm} \pm 0.0004 \mathrm{~nm} \\
0.041^{\circ} \pm 0.001^{\circ}\end{array}$ \\
\hline $\begin{array}{l}\text { Effective area } \\
\text { (this work) }\end{array}$ & $\begin{array}{c}0.30 \pm 0.03 \mathrm{~cm}^{2} @ 125 \mathrm{~nm}, 0.15 \pm 0.02 \mathrm{~cm}^{2} @ \\
140 \mathrm{~nm} \text {, and } 0.05 \pm 0.01 \mathrm{~cm}^{2} @ 160 \mathrm{~nm}\end{array}$ \\
\hline
\end{tabular}

Note. Information gathered from Gladstone et al. (2017a), Greathouse et al. (2013), and this work.

\subsection{Instrument Orbital Operation}

The versatility available in Juno-UVS commanding makes it usable for a wide range of observations. During much of each orbit Juno-UVS is off. When on, the different observations performed are, typically

1. perijove observation,

2. synoptic observation,

3. calibration observation,

4. and radiation monitoring.

Perijove observation. The perijove observations are nominally performed for about $10 \mathrm{hr}$ centered on perijove. The spatial resolution on Jupiter changes drastically during this period, and extensive use of the scan mirror is used to target different auroral features on Jupiter. The strategy in these is to optimize the data acquired, while avoiding high background radiation regions. Radiation models are used in order to avoid as much as possible taking data in the high radiation regions (Becker et al. 2017). The interested reader can refer to the work of Kammer et al. (2018) for further information on the strategies adopted to mitigate the impact of the radiation on the science return.

Synoptic observation. The synoptic observations are performed when Juno is located in the outer part of the orbit. During these observations, Jupiter appears as $\sim 1^{\circ}$ to UVS meaning the instrument can still resolve the disk, but only with a few resolution elements making these observations useful for auroral power calculations rather than auroral imaging studies (e.g., Gladstone et al. 2017b). Since the plane of Juno's orbit is inertial, the orientation of that plane evolves from a dawn/dusk orbit (PJ1) to a midnight/noon orbit (PJ22) due to the orbit of Jupiter about the Sun. This evolution means that UVS does not have the capability to observe Jupiter in the synoptic configuration from PJ10 (2017 December 16) up to $\sim$ PJ33 (2021 April 15). Since PJ10, regular half-sky scans have been performed instead of the synoptic observations, where the full range of the scan mirror is used in order to map half of the sky.

Calibration observation. Calibration observations are performed regularly to evaluate the health of the instrument. Four scan mirror pointings are chosen for the calibration observations so that $\sim 2.5 \mathrm{hr}$ of time may be spent at each pointing, depending on the instrument's parameters (HVPS). For each swath of the spacecraft, a given point source is observed during typical $\sim 17 \mathrm{~ms}$ and $\sim 2 \mathrm{~ms}$ integration times through the wide and narrow slits, respectively. This gives an effective integration time on any one star of $\sim 4 \mathrm{~s}$ for the wide slit and $0.6 \mathrm{~s}$ for the narrow slit, when $2.5 \mathrm{hr}$ of observations are coadded. See the work of Greathouse et al. (2013) for a more indepth discussion.

Radiation monitoring. Finally, the radiation observations are performed to monitor the radiation environment by running the instrument with the aperture door closed and by recording the events triggered by high-energy electrons, gamma-rays, and galactic cosmic rays passing through the instrument shielding. Figure 2 represents a typical orbital sequence performed during PJ8 (2017 September 1). 


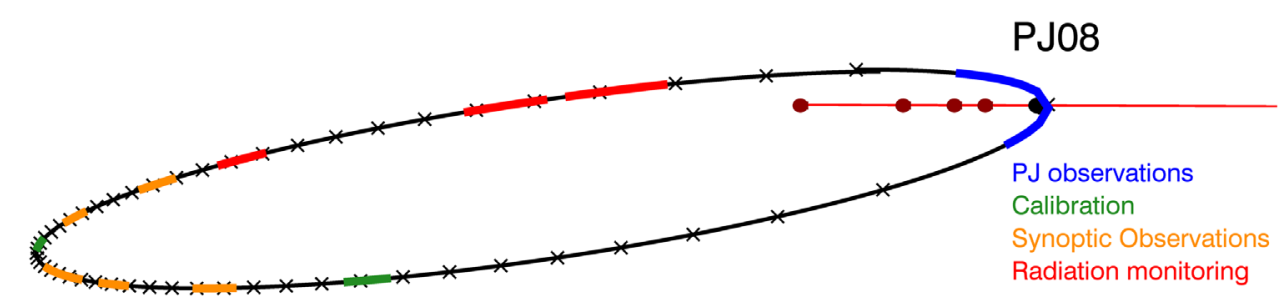

Figure 2. Overview of the UVS observations during PJ8. The straight horizontal red line represents the orbital plane of the Galilean satellites, which are denoted by the red dots. Jupiter is located at the center of the horizontal line. Each tick mark correspond to a one day increment with respect to the perijove date.

Table 2

Time Period of the Main Cruise Events Discussed in the Text

\begin{tabular}{lll}
\hline \hline Phase & Event & Time Period \\
\hline Launch & & 2011 DOY 217 \\
Commissioning & High-voltage commissioning & 2011 DOYs 339-347 \\
Cruise & Periodic maintenance 1 & 2012 DOYs 294-297 \\
Earth flyby & Earth flyby observations & 2013 DOYs 273-283 \\
Cruise & ISON comet observation & 2013 DOYs 308-2014 DOYs 039 \\
Cruise & Periodic maintenance 2 & 2014 DOYs 115-118 \\
Cruise & Periodic maintenance 3 & 2015 DOYs 100-105 \\
Jupiter approach & Approach phase (JC59-JC63) & 2016 DOYs 025-131 \\
Jupiter approach & Approach phase (JC64) & 2016 DOYs 155-182 \\
Jupiter orbital operation & Jupiter orbital insertion (JOI) & 2016 DOY 187 UTC 02:47:32 \\
\hline
\end{tabular}

\section{In-flight Characterization of Juno-UVS}

\subsection{Overview}

An inspection of the total number of events recorded by UVS gives a measure of how many UV photons and radiation events the instrument has experienced since its launch. Figure 3 summarizes the fluence experienced by the instrument since its launch. This quantity was calculated as

$$
\Phi=\frac{\epsilon \times e \times \sum \mathrm{ACs}}{\sigma_{\mathrm{MCP}}},
$$

where $\Phi$ is the charge depletion, i.e., the amount of charge extracted from the glass plates due to photon counting, in $\mathrm{C} \mathrm{cm}^{-2}, \epsilon$ is the overall detector gain $\left(\epsilon=2 \times 10^{7}\right.$, from Gladstone et al. 2017a), and $\sigma_{\mathrm{MCP}}$ is the area of the MCP $\left(3.5 \times 1.8 \mathrm{~cm}^{2}\right)$. The last term of the right-hand side of the equation represents the summation over the number of events recorded in the analog list, i.e., the analog counts (ACs).

The charge depletion is presented here per data set to provide an inter-data set comparison. It does not represent the total charge depletion experienced by the detector, which can instead be computed by the cumulated charge depletion since launch. During cruise, from launch up to mid-2016, UVS performed regular routine observations, such as periodic maintenances, HVC, or dedicated observations (e.g., Earth flyby, observations of the ISON comet). The different types of observations are color coded and dark green denotes the JC64 approach sequence that ran for 26 days straight. The time period corresponding to each of the event discussed are presented in Table 2.

Overall, the amount of charge depleted per data set stays fairly constant during the cruise to Jupiter, around $5 \times 10^{-5} \mathrm{C} \mathrm{cm}^{-2}$, with quite a large scatter. These variations are related to differences in the pointings used and the decrease of the Ly $\alpha$ interplanetary medium (IPM) background as Juno travels farther from the Sun (Broadfoot et al. 1989).

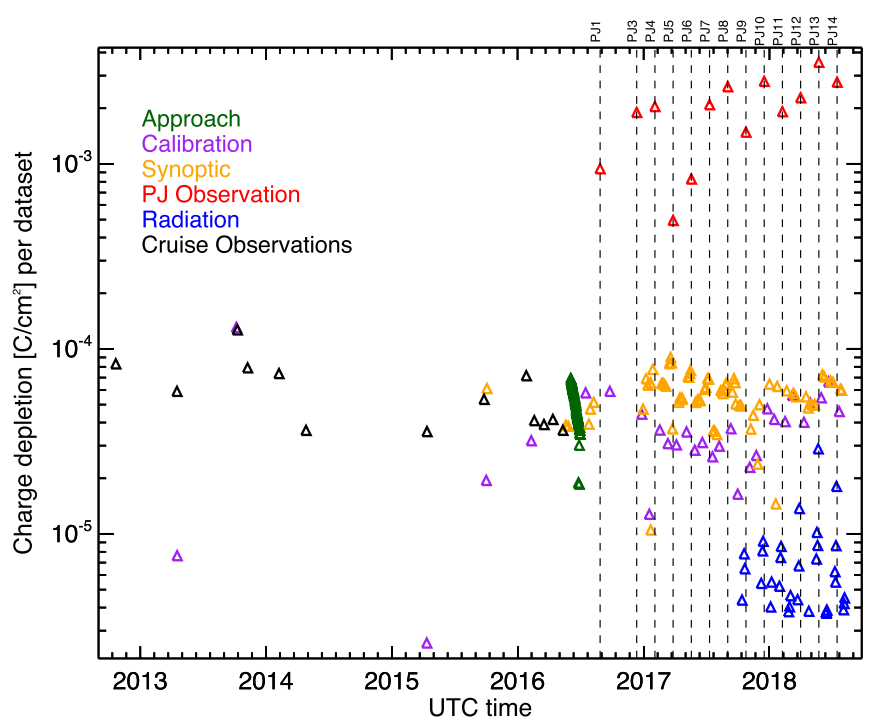

Figure 3. Overview of the charge depletion experienced by UVS, broken down by recorded data set, from launch until PJ11. The different types of observation are color coded. All of the synoptic observations recorded past PJ10 are actually half-sky scans due to the limited visibility of Jupiter away of PJ (see Section 3.1 for more details).

The JC64 sequence was a special sequence of almost continuous observation of Jupiter during approach, taken from 2016 June 3 to June 29 (Gladstone et al. 2017b). It used a small set of scan mirror pointings to keep Jupiter well centered in the UVS lower wide slit. The variation in the charge depletion over the JC64 observation period cannot be attributed to variation of the Ly $\alpha$ IPM background since the spacecraft/Jupiter/Sun geometry was nearly constant over this period. However, the overall count rate was measured to have decreased by a factor of 2 over this 26-day-long observation period, mostly due to scrubbing and loss of sensitivity at Ly $\alpha$. 


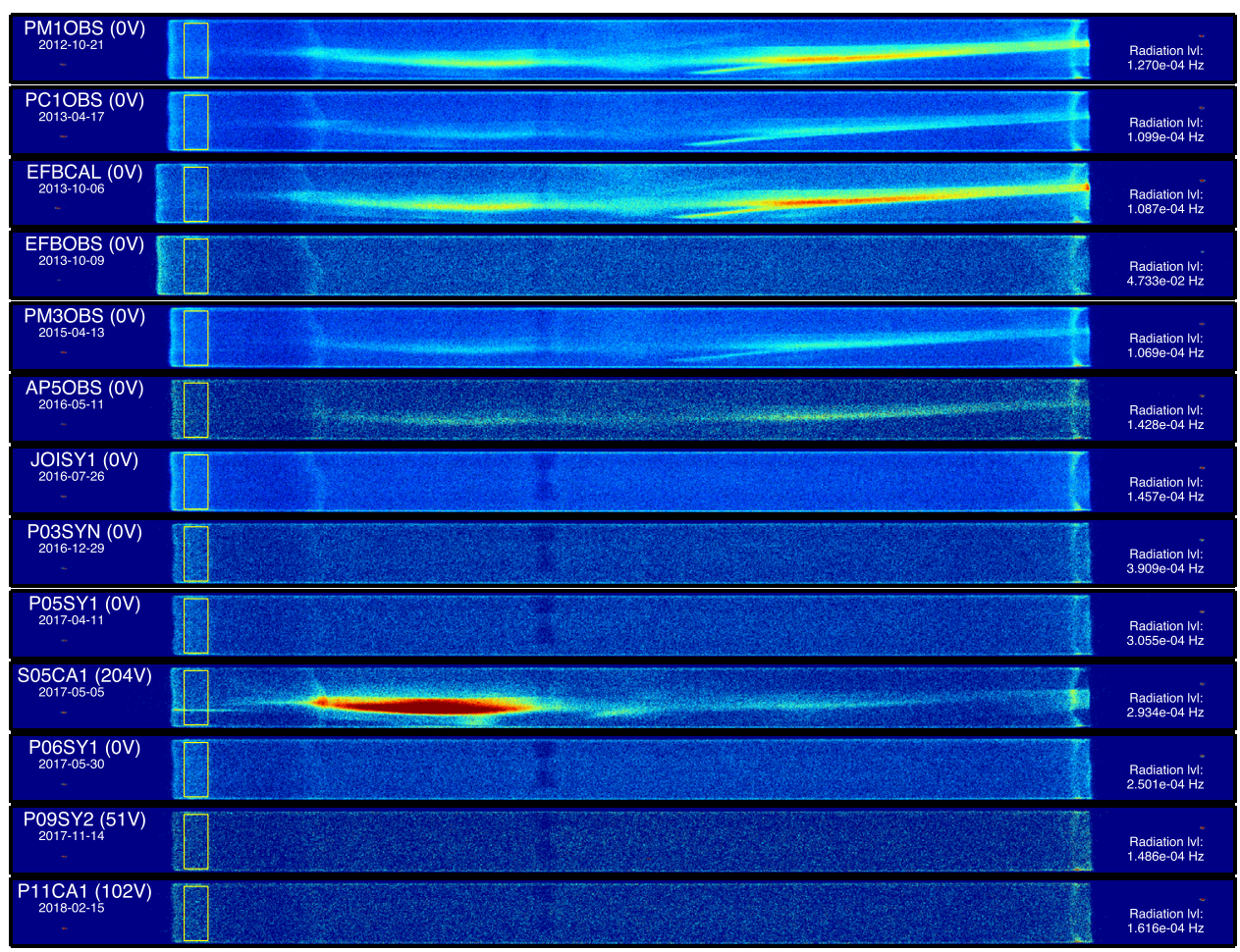

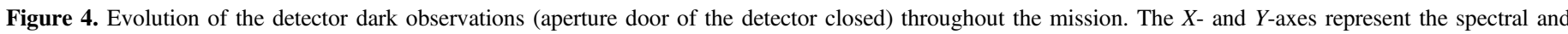

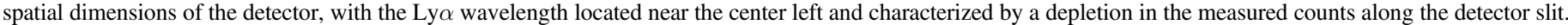

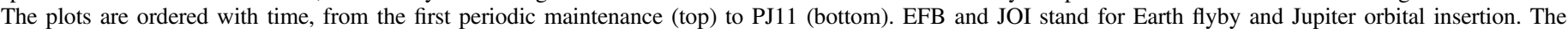

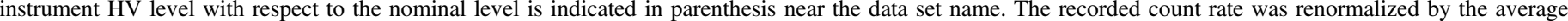
count rate measured within the yellow box. These numbers are written on the left-hand side of each panel.

Once at Jupiter, the orbital operations clearly show extensive use of the detector. The charge depletion of the detector during the PJ observations is $\sim 40$ times that of any other observation performed. The large scattering of the PJ observation points are mostly due to the orientation of Jupiter's magnetic field, which controls the amount of counts UVS receives due to high-energy electrons.

Numerous dark observations have been performed throughout the mission. They consist of running the instrument with the aperture door closed, and provide an assessment of the detector performance. Such an evaluation of the detector performance through selected dark observations is presented in Figure 4 . The background level experienced by the spacecraft may vary during each dark observation. Each dark observation in Figure 4 has been normalized by the averaged count rate within the yellow box shown on the left-hand side of the detector.

Previous work showed that the detector dark counts exhibited a long arcuate feature early in the mission, although it was thought not to cause problems given its low count rates compared to observational sources of interest (Greathouse et al. 2013). The feature disappeared during the Earth flyby observation data set, possibly drowned out by the high level of radiation experienced. It then reappeared during cruise and definitely disappeared once into the Jovian system. Another likely related feature briefly appeared when the potential difference of the HVPS was increased for the first time at $-204 \mathrm{~V}$ below the nominal HVPS level, during a calibration observation taken around PJ5. The new feature was observed during one calibration data set, and never reappeared later (as of PJ14), even when testing the instrument response at a higher HVPS level.
The depletion in detector counts seen at $\operatorname{Ly} \alpha$ is characterized by the vertical hourglass that stands out of Figure 4. Located near the center left of the detector spectral range, it progressively shows a dip in the number of counts recorded at these wavelengths, and clearly reveals the shape of the slit during the Jupiter operation.

\subsection{Monitoring of the Health of the Instrument}

The extensive use of UVS requires close monitoring of the health of the instrument. Numerous stars have been used throughout the mission as calibration standards to inspect the sensitivity of the instrument as a function of time.

For example, Figure 5 presents several UVS calibration spectra of the UV-bright star A Pup (HD 54893). This star was observed multiple times over more than $3 \mathrm{yr}$ through the wide part of the slit, at the nominal HV level $(-4177.5 \mathrm{~V})$. Only the 2014 measurements shows sensitivity to $\operatorname{Ly} \alpha$. The right-hand side of the plot shows the PHD of the photons recorded for every visit, with the Ly $\alpha$ region and longer wavelengths separated. A minor design flaw in the detector electronics causes pulse heights that would otherwise fall in bins 17-32 to instead all stack up in bin 17, without affecting the counting efficiency. This flaw is the reason some PHDs show a large spike in bin 17. In order to be able to detect a photon, the pulse height triggered by a photon must lie within the range of the discriminator. After an extensive use of the instrument, the glass plates are scrubbed of charge in the areas of highest integrated fluence, making future events measured in these areas have a lower pulse height, also called gain sag. This phenomenon can be tracked by monitoring the PHD over time. At the nominal HV level, UVS lost its sensitivity to Ly $\alpha$ 

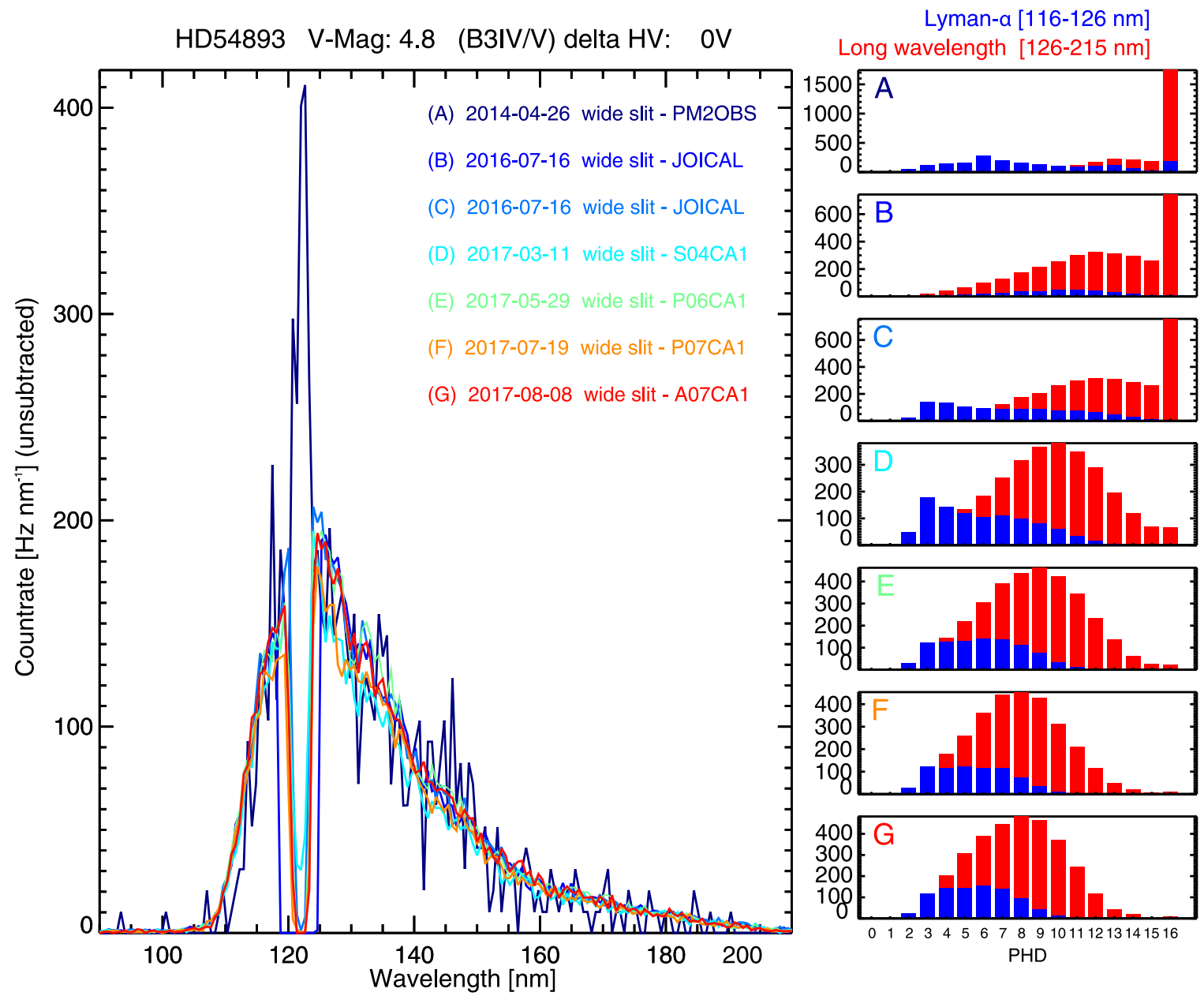

Figure 5. Left panel: evolution of the count rate measured by Juno-UVS for the star HD 54893 (A Pup) at the nominal HV level from 2014 April to 2017 August. Measurements were done through the wide part of the slit. Right panel: evolution of the pulse height distribution (PHD) measured by Juno-UVS corresponding to every single measurement (see Section 2.1 for more details about the PHD). The blue color denotes the Ly $\alpha$ (116-126 nm) while the red denotes the longer wavelength (126-215 nm).

through the wide slits prior to reaching Jupiter. UVS is however still sensitive, as of PJ14, at the longer wavelength photons and through its wide and narrow slits. As the mission progressed, the PHD of longer wavelengths were observed to gradually shift to lower bins. In order to avoid losing sensitivity at the longer wavelength, the $\mathrm{HV}$ of the instrument was regularly raised over the mission. The reader interested in the effect of gain sag can refer to the work done on, e.g., the Hubble Space Telescope Cosmic Origins Spectrograph (HSTCOS; Osterman et al. 2011; Sahnow et al. 2011).

Note that the UVS settings for the curve B of Figure 5 were such that the instrument was masking the Ly $\alpha$ region. Note also that, although HD 54893 was included in the General Catalog of Variable Stars (GCVS; Samus' et al. 2017), it was ranked as a suspected variable (Kholopov 1982). A closer look to the published observations shows little to no variations (Jerzykiewicz \& Sterken 1977).

\subsection{Quantification of the Sensitivity Loss}

Due to the extensive sky coverage, long periods of time away from PJ, and large available downlink, Juno-UVS is able to perform extensive tracking of instrument sensitivity. This is accomplished by looking at a handful of stars throughout the mission, over a wide range of HV values and well-sampled

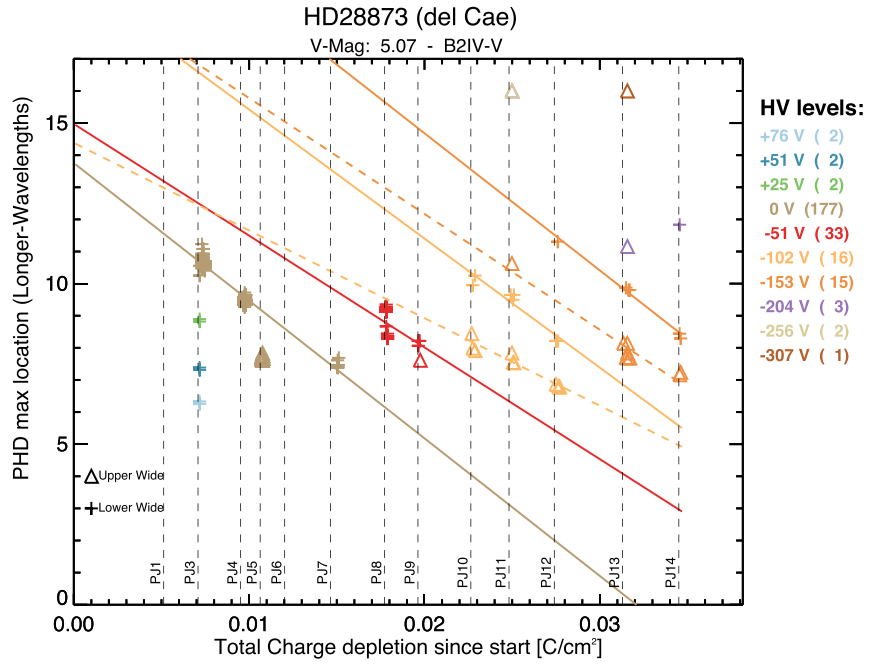

Figure 6. Evolution of the PHD peak location as a function of the total charge depletion since launch, for the star HD 28873 (del Cae). Measurements made at different voltage levels are shown with different colors according to the legend, along with the corresponding number of measurements in parenthesis. Measurements made through the lower wide slit are shown with plus signs, while upper wide slit measurements are shown with triangles. The linear fits were performed per voltage setting and per slit. Lower and upper wide slit fits are shown with solid and dashed lines. 


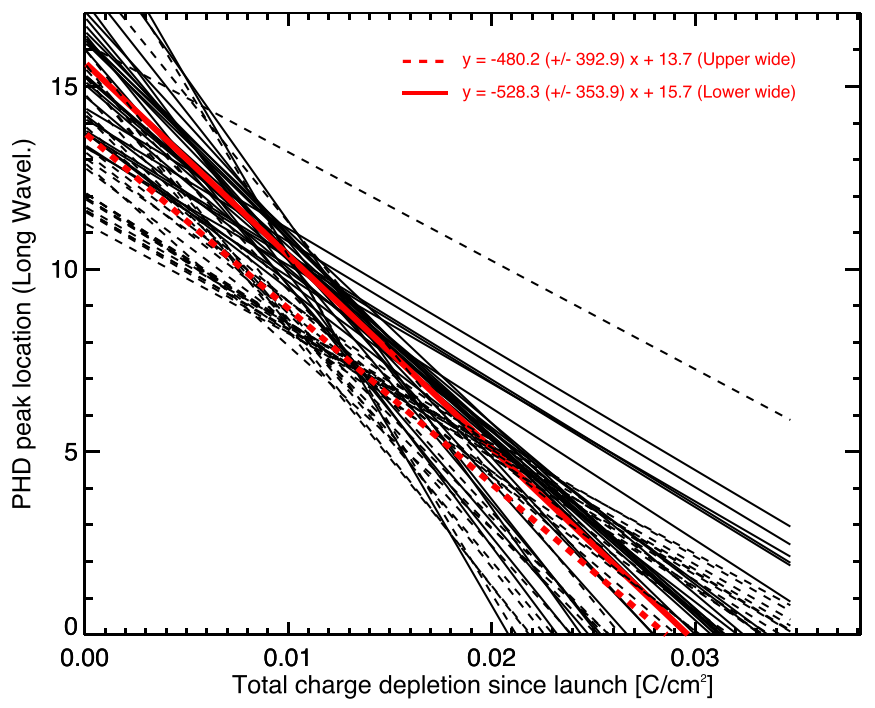

Figure 7. Representation of the linear fits performed on the PHD peak location, for stars detected at the nominal HV level in the lower wide slit (solid lines) and the upper wide slit (dashed lines). The averaged linear trends are shown in red.

temporal cadence. To illustrate, Figure 6 shows how the location of the PHD peak evolves as the mission progresses. Since the sensitivity of UVS is expected to decrease with the instrument usage, this was plotted as a function of cumulative charge depletion since launch, making the $x$-axis nonlinear in time. The star observed was HD 28873 ( $\delta$ Cae), a 5.07 $V$-magnitude B2IV-V star, for which only the wide slit measurements are shown here. The cumulative charge depletion at the time of each $\mathrm{PJ}$ is also represented through the vertical dashed line. Since the fluence experienced by UVS varies at each PJ (see Figure 3), the PJ positions are not equally distributed in the cumulative charge depletion space.

HD 28873 was observed over a wide range of HV levels, displayed by the labels on the left-hand side of the graphic, and showing the difference in the HV level with respect to the nominal one. The numbers reported in parenthesis next to the $\mathrm{HV}$ level denote the number of measurements performed at that HV setting. Most of the observations have been performed at the nominal HV setting. Shortly after PJ3, several calibration observations at a lower voltages were performed in order to quantify the instrument sensitivity at these $\mathrm{HV}$ settings. Consequently, the PHD was shifted toward the lower discriminator level. Calibrations at several higher voltage settings above the nominal one were also performed throughout the mission to pre-test the instrument response at future settings. The FWHM of a healthy PHD is about 5 bins. In order to optimize the detection efficiency, the UVS parameters should remain such that the PHD maximum does not go lower than bin number 5-8. Consequently, the potential difference of the HVPS of UVS was increased by $-51 \mathrm{~V},-102 \mathrm{~V}$, and $-153 \mathrm{~V}$ below the nominal HV level prior to PJ8, PJ10, and PJ13, respectively. Proceeding to these changes hence increases the absolute value of the voltage applied, keeping the instrument detection efficiency steady over time.

The PHD peak location of the upper wide slit data points are systematically shifted down by $\sim 1-2$ bins, for a measurement over the same star at a given time and voltage setting. Consequently, data points corresponding to lower wide slit measurements are shown by plus signs, while upper wide slit measurements are shown by triangles. The colored straight

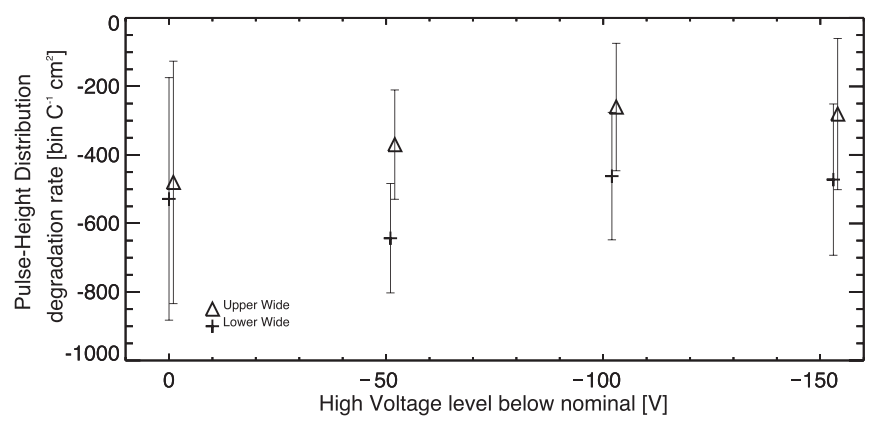

Figure 8. Evolution of the pulse height distribution depletion rate of the UVS detector, at several high-voltage settings above the nominal high-voltage level. The depletion rate for the upper and lower wide slits are shown with triangle and cross symbols. The uncertainty on the rates are taken as the FWHM of the individual depletion rates distribution from Figure 7.

lines represent fits of the PHD peak location as a function of charge depletion, at a given HV setting. The fits on the lower and upper wide slit measurements, when enough data was available, are shown with solid and dashed lines.

This operation was performed for a number of stars that fall into a reasonable range of magnitude ( $V$-mag between 4.5 and 6.5 ), and that have been detected multiple times at nominal voltage. The position of the PHD peak was tracked for all of these stars and fitted as a function of charge depletion, for a given voltage setting. At the nominal voltage level, 46 stars fulfilled these criteria: 31 stars were measured through the lower wide slit and 28 stars through the upper wide slit. Figure 7 shows how the PHD peak location decreases over time for this subset of stars. Measurements made through the lower wide slit are shown with solid lines, while upper wide slit measurements are shown with dashed lines. Several outliers have been removed by excluding the extremum of the fits (i.e., minimum and maximum values for the slopes of the lines). The overall charge depletion between the two wide slits seems consistent with one another, given the scatter on individual star charge depletion measurements. Note that the upper wide slit should be used first to track down the moment where the detector will start missing photons, since it exhibits an offset in term of PHD peak location of about 1.7 bins.

The rate of charge depletion (i.e., the slope in Figure 7) can be compared for the measurements made at different voltage settings. Figure 8 represents the PHD depletion rate for the three voltage settings used during the PJ observation, as of PJ14. The depletion rate experienced by UVS at different HV levels stays constant for the different settings, within uncertainties.

\section{In-flight Calibration of Juno-UVS}

In this section, the method developed to calibrate Juno-UVS is described. Since Juno-UVS cannot sit and stare at welldefined and calibrated stars, we decided to use the extensive sky coverage as a strength by extracting a large number of stellar spectrum. We then compare them with previous observations performed at overlapping bandpasses. In this section, we first describe the stellar spectrum databases used to calibrate the instrument. Then, we describe how the effective area of the instrument was retrieved.

\subsection{Definition of the Stellar UV-spectra Database}

Observations made by two UV observatories have been used as a reference: the International Ultraviolet Explorer (IUE) and the 
Hubble Space Telescope (HST). The HST observations used are part of the Space Telescope Imaging Spectrograph (STIS) Next Generation Spectral Library ${ }^{5}$ (Heap \& Lindler 2010; Lindler \& Heap 2010), as well as observations compiled in the CALSPEC $^{6}$ library.

One of the most extensive stellar UV-spectral databases comes from the IUE mission. It recorded over 100,000 UV spectra between 1978 January 26 and 1996 September 30. The satellite observed a variety of astrophysical bodies, ranging from comets, planets, stars, and supernovae to galaxies, in the $115-335 \mathrm{~nm}$ range, at high $(0.01-0.03 \mathrm{~nm})$ and low $(0.6-0.7 \mathrm{~nm})$ spectral resolution.

The IUE spectral database was the prime source used to calibrate Juno-UVS. All of the spectra recorded by IUE for O, $\mathrm{B}$, and A stars were downloaded from the MAST database, ${ }^{7}$ leading to a total of about 45,000 different spectra. A closer inspection at specific stellar spectrum shows a great variability between measurements recorded of the same star. The IUE database, which includes a large number of observations taken at different times, with different observing modes and instrument parameters, was refined in order to only keep the spectra that were thought to be reliable and usable for calibration.

The IUE had two spectrographs, the short-wavelength spectrograph, operating between 115 and $200 \mathrm{~nm}$, and the longwavelength spectrograph, operating between $185-335 \mathrm{~nm}$. Two cameras per spectrograph were developed, the prime and redundant cameras for the short-wavelength spectrograph (SWP and SWR, respectively), and similarly for the long-wavelength spectrograph (LWP and LWR). The SWR was not fully functional during the mission, and no spectra recorded with it were used within our process. IUE was capable of observing sources through two different apertures: the large aperture $\left(\sim 10^{\prime \prime} \times 20^{\prime \prime}\right.$ ellipse $)$ and the small aperture $\left(\sim 3^{\prime \prime} \times 3^{\prime \prime}\right.$ circle $)$. The size of the image for a point source in the aperture plane was $4^{\prime \prime} \times 4^{\prime \prime}$, making the absolute flux calibration of the small-aperture observations difficult (Garhart et al. 1997; Massa \& Fitzpatrick 2000). Consequently, only the large-aperture observations were used. IUE data recorded under abnormal conditions were not used. Several flags in the FITS headers were written to report these anomalies. Therefore, the IUE fits files recorded with at least one of these header flags written as affirmative value (i.e., YES) were discarded: ABNBADSC, ABNHISTR, ABNHTRWU, ABNMICRO, ABNMINFR, ABNNOSTD, ABNOTHER, ABNREAD, and ABNUVC. The spectra of variable stars that were found in the GCVS (Samus et al. 2017) were also discarded. The International Ultraviolet Explorer Data Analysis Center (IUEDAC) IDL Software Libraries ${ }^{8}$ were used to read in the IUE fits files, which involve the New Spectral Image Processing System (NEWSIPS) calibration pipeline (Nichols \& Linsky 1996). Further flux calibration correction was applied on the low-dispersion data, following the investigating from Massa \& Fitzpatrick (2000). Only the observations recorded using a single exposure mode were used. The observations recorded with the trailed exposure mode were discarded, as well as the ones designed with an offset in the $X-Y$ axis of IUE's Fine Error Sensor (FES; see Garhart et al. 1997).

\footnotetext{
5 https://archive.stsci.edu/prepds/stisngsl/

6 http://www.stsci.edu/hst/observatory/crds/calspec.html

7 https://archive.stsci.edu/iue/search.php

8 https://archive.stsci.edu/iue/iuedac.html
}

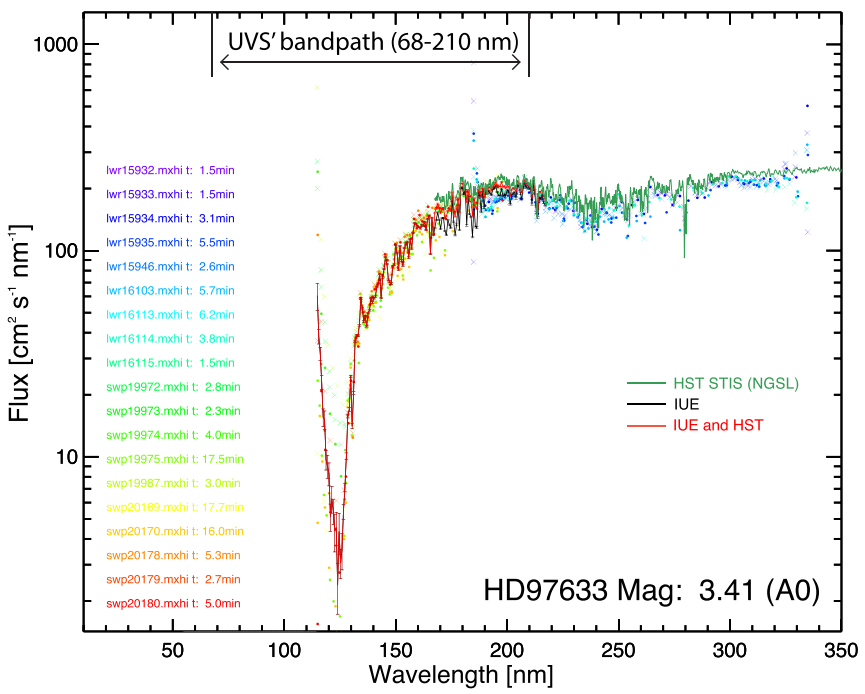

Figure 9. Illustration of the definition of the stellar UV-spectra database for HD 97633. IUE observations are shown by the dot and cross symbols. Crosses denote the $I U E$ data excluded in the refinement algorithm (see the text for more details). The labels on the left side list the IUE filename plotted here, along with their respective exposure time. The green solid line shows the HST observations. The black and red solid lines represent the resulting spectra averaged over the selected $I U E$ data points, and mean $I U E+H S T$ combined spectrum, respectively, degraded to the UVS spectral resolution.

A recent correction for the IUE low-dispersion flux calibration was recently published by Bohlin \& Bianchi (2018) to better match the CALSPEC library. We closely compared the refined IUE database using that correction with the CALSPEC and NGSL database. Although it seems clear that this correction provides a better match of the IUE observations with the CALSPEC library, the agreement between the IUE and NGSL however degraded at the same time. In this first version of the Juno-UVS calibration, the correction published by Bohlin \& Bianchi (2018) is not accounted for.

When applying these selection criterion, the remaining IUE database used for calibration totals about 1800 different stars. Some of them have been observed multiple times, and, by crosscomparing the spectra for the given stars, several measurements still exhibited discrepancies and/or significant variabilities. For instance, the measurements showed systematic discrepancies in the outermost part of the spectrograph bandpass, particularly the LWP/LWR spectrographs. We limited the bandpass of the SWP spectrograph to be 115-200 nm (Nichols \& Linsky 1996), and the LWP/LWR spectrographs to be $185-335 \mathrm{~nm}$ (Massa \& Fitzpatrick 2000).

In order to build a catalog of stellar measurements, we degraded this refined IUE database to the Juno-UVS resolution. Further refinements have been applied (i) when three or more measurements of the same star have been recorded by excluding the IUE measurements that appeared statistically different from others, (ii) by discarding measurements recorded with the shortest exposure time, (iii) by cross-comparing the IUE spectrum in the overlapping spectral region of the SWP and LWP/LWR and discarding the measurements that appeared significantly off, (iv) by discarding single measurements presenting a large gap in wavelength, and (v) by crosscomparing with $H S T$ observations.

When three or more IUE measurements taken with the same spectrograph (SWP or LWP/LWR) were recorded, the averaged flux and standard deviation in several specific 

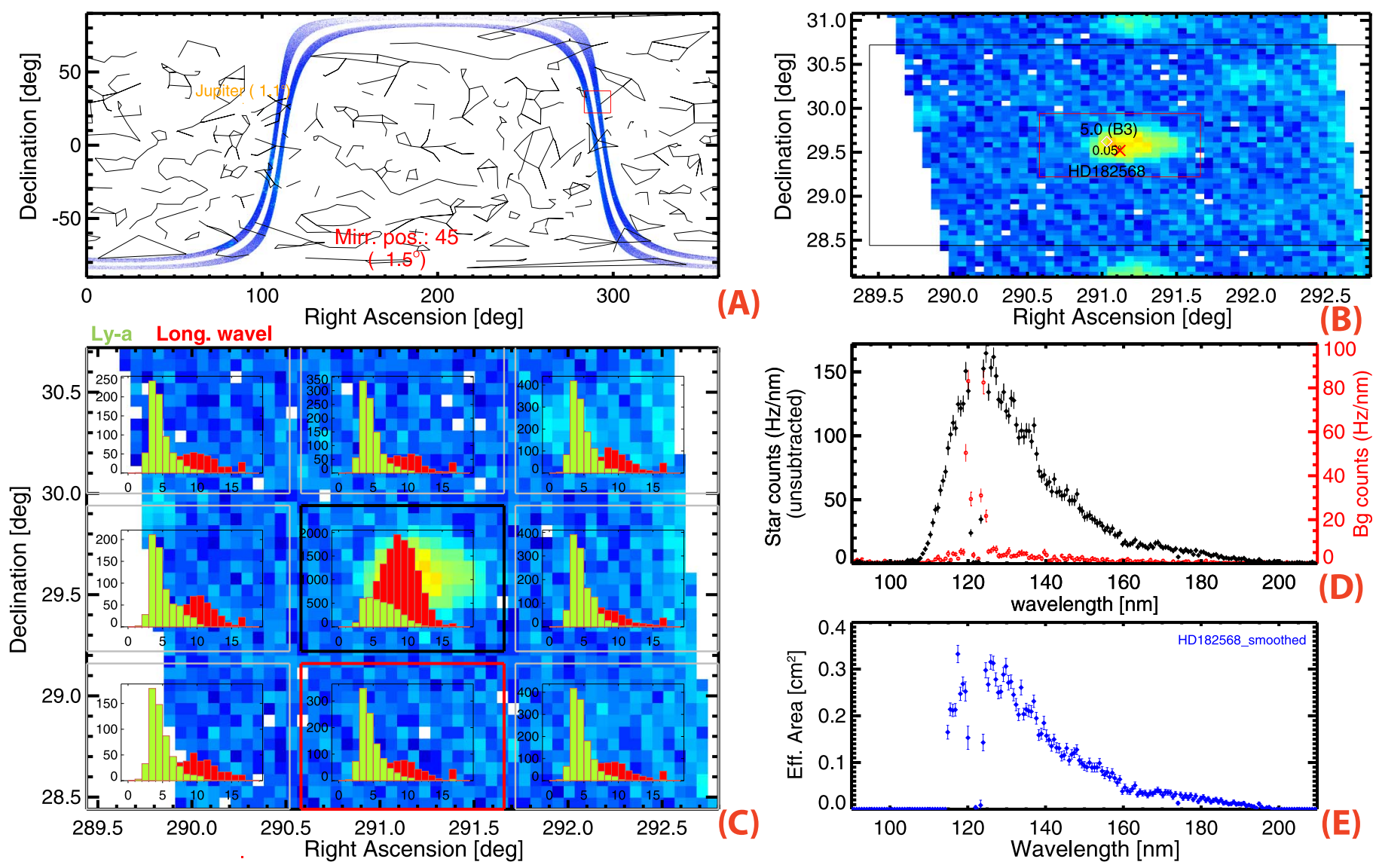

UVS expo: $4.761 \mathrm{~s}$ (tot. time: $2.379 \mathrm{hrs)} \quad$ UVS HVPS: $-4177.49 \mathrm{~V}$ Frames: 35 to 370 Acq. tag: 34272 Detector row: 203 (wide) 2017 MAR $11 \quad$ Tot. counts: $19296.74(4053.27 \mathrm{~Hz})$ Dataset: UVS_542527417_2017070_S04CA1_V01_012 jm0042

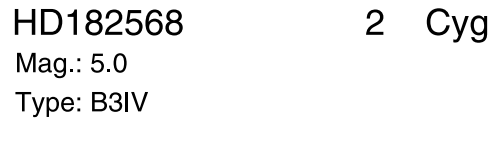

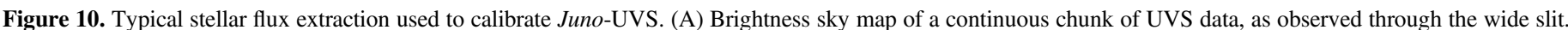

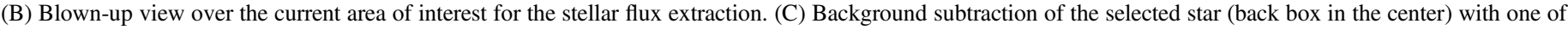

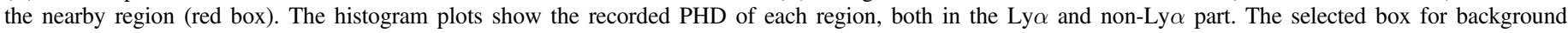

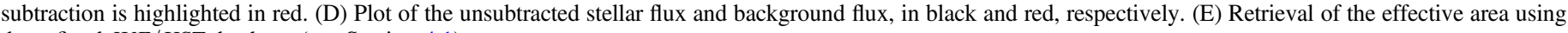
the refined IUE/HST database (see Section 4.1).

wavelength regions were computed. Measurements further apart than one standard deviation have been discarded. When five or more measurements with the same instrument were recorded, the bottom $15 \%$ in terms of exposure time duration have been discarded. When both short- and long-wavelength measurements for the same star exist, the averaged flux in the overlapping spectral region of $190-195 \mathrm{~nm}$ were used to discard the long-wavelength measurements that were $\sim 10 \%$ or more different from their short-wavelength counterpart. Several measurements presented a large gap in terms of wavelength coverage and they were similarly discarded. Finally, HST observations were used to cross-compare the IUE observations in the $170-185 \mathrm{~nm}$ region. When the relative difference with the IUE observations exceeded $80 \%$, only the HST observations have been retained for the calibration. An example of the IUE observation selection process is illustrated in Figure 9.

\subsection{Calculation of the Effective Area}

In this section, we describe how the effective area of UVS has been retrieved using the IUE/HST spectra database established previously. As shown in Figure 3, most of the instrument sensitivity at $\operatorname{Ly} \alpha$ in the wide slits was lost prior to JOI. We will first discuss how the non-Ly $\alpha$ calibration was performed, and then we briefly discuss the Ly $\alpha$ calibration.

\subsubsection{Non-Ly $\alpha$ Calibration}

Because Juno is a spinning spacecraft that nominally spins at $2 \mathrm{rpm}$, it is not possible to sit and stare at specific stars known to be reliable for calibration purposes. Instead, the mirror position is defined such than Juno-UVS will observe a handful of stars over one spin. Several spins of data recorded at the same mirror position were co-added to increase the signal-tonoise ratio. During each spin, a point source will be observed for about $17 \mathrm{~ms}$ in the wide part of the slit and $1.9 \mathrm{~ms}$ in the narrow part of the slit, when the mirror position is nominal, i.e., when the UVS boresight direction is within the spin plane. The integration timescales up as $1 / \cos ^{2} \alpha_{i}$, where $\alpha_{i}$ is the angle between pixel $i$ along the slit and the Juno spin planethe square of the cosine is due to both the circle described on the sky being smaller and the star crossing the slit at an angle. 


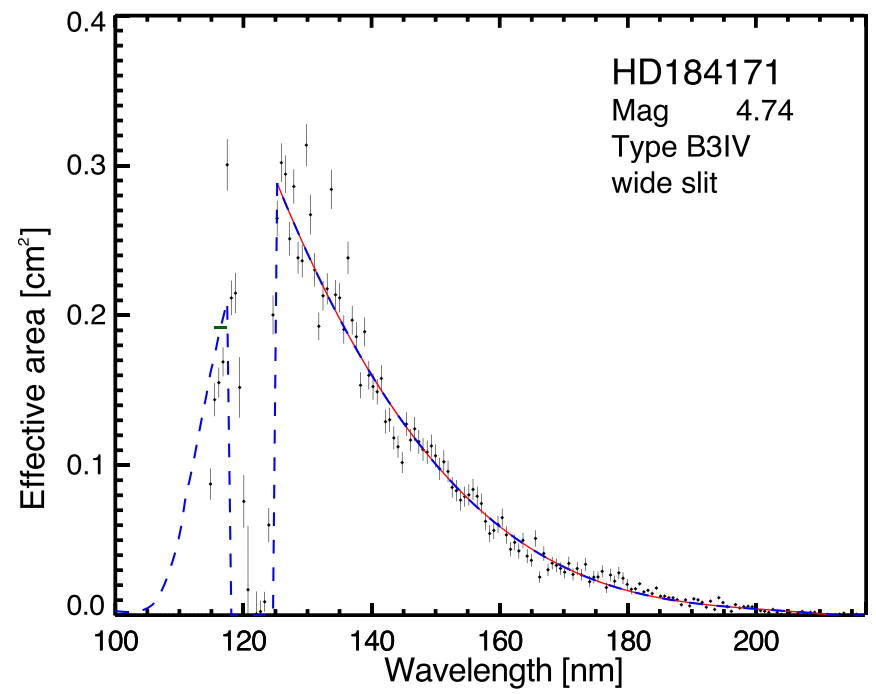

Figure 11. Illustration of the fit performed on a given effective area measurement. The star recorded was HD 184171, a B3IV star with 4.74 $V$-magnitude, through the wide slit.

The following calibration algorithm has been written into an automated IDL calibration code and is illustrated in Figure 10. One single continuous chunk of the Juno-UVS observation is first considered, implying that it was recorded with the same instrument parameters without any temporal gaps and at a single mirror position. The results are mapped and displayed in Figure 10(A). The routine starts with the brightest star in the map and moves toward dimmer stars as it proceeds. Several tests are performed on the selected stars to assess whether they are good for calibration (see Figure 10(B)). If it fails any of the tests, the star is masked and the algorithm proceeds to the next one. These tests range from checking that the stars do not have an abnormal size by fitting a Gaussian, was not recorded too near the ends of the slit, is not too close or merged to other bright stars, and finally that it can be attributed to a known star within a certain angular distance from the detected centroid. Once the star passes these tests, the background subtraction is done by finding a nearby region, devoid of light contamination from neighboring stars, preferentially recorded in a detector region that is as close as possible from the one of the considered star (see Figure 10(C)). Results of the extracted stellar and background fluxes are shown on Figure 10(D), in black and red, respectively, and correspond to the black and red boxes of Figure 10(C). Finally, if a measurement exists for that star in our refined IUE/HST observations, the effective area of Juno-UVS is calculated (Figure 10(E)).

That algorithm is automatic and generates typical plots such as the one in Figure 10 for all detected stars. It has been run on all the synoptic and calibration observations (recall Figure 2). Up to PJ14 (2018 July 16), around 8700 individual stellar spectra have been extracted, 7500 and 1200 through the wide and narrow slits, respectively. Many stars have been observed multiple times. Only spectra for which previous $I U E / H S T$ observations exist have been retained, meaning that the effective area of the instrument has been retrieved that same number of times.

Bright stars cause localized gain suppression on the detector when observed through the wide slits, which makes the detector miss a fraction of the incoming photons. Only a subset of stars can be used for calibration. Furthermore, since the throughput of the wide slit versus the narrow slit is different, the range of the $V$-magnitude of the stars retained for calibration depends on the slit considered. Previous work of Greathouse et al. (2013) used only a handful of stars to perform an early calibration during the HVC. They used stars with a $V$-magnitude around 3.15-4.7 and around 2-2.5 for the wide and narrow slits, respectively. In this work, we used stars in the $V$-magnitude range of 4-6 and 1-3 for the wide and narrow slits, respectively.

Every retained effective area measurement was subsequently fitted following the procedure described on Figure 11. We fitted the $125-220 \mathrm{~nm}$ region with a third order polynomial function. The 115-119 nm region was found challenging to fit due to large uncertainties on the measurements. In addition, there is a lack of reliable calibration data at wavelengths shorter than $119 \mathrm{~nm}$ over the large set of stars observed by UVS. We fitted the wavelength shorter than $119 \mathrm{~nm}$ using a theoretical effective area expected and rescaled to fit the mean measured effective area in the $115-119 \mathrm{~nm}$ range. The theoretical effective area was calculated considering the optical design of Juno-UVS and assuming an $\mathrm{MgF}_{2}$ coating on all of the optics (Gladstone et al. 2017a). Using a subset of the brightest stars detected by UVS, we also compared our effective area calculation at these short wavelengths where the extrapolation is done using Kurucz models (Kurucz 1979). Our results are in very good agreement considering the measurement uncertainties, and the artificial nature of the calibration. The same exact procedure was performed on the measured errors.

This procedure was performed for all of the effective area measurements that fulfilled the aforementioned criteria. Figure 12 provides a summary on the overall retrieved effective areas as a function of time and $\mathrm{HV}$ setting. Each column denotes a different part of the slit: lower wide slit (left), narrow slit (middle), and upper wide slit (right). Each row displays an ensemble of the retrieved effective areas at a given $\mathrm{HV}$ level and a given time. The number of measurements used for each panel is shown in parenthesis. While the white solid lines denote the mean effective areas, averaged from every single fitted effective area measurements (recall Figure 11), the white dashed and dotted lines were calculated using these 2D histogram distributions by finding the center and the standard deviation of the distribution for each wavelength bin.

Figure 12 suggests than that are no obvious changes in the instrument sensitivity at non-Ly $\alpha$ wavelengths across the detector and over time. A closer inspection on a time series of the upper wide slit retrieved effective areas, i.e., the one that could potentially first shows signs of gain loss, is presented in Figure 13. Given the uncertainties on the effective area measurements, the upper wide slit does not exhibit any temporal variability in the retrieved effective area when compared to the averaged one.

\subsubsection{Ly $\alpha$ Calibration}

A procedure similar to the one used for the non-Ly $\alpha$ region was carried out here, with a few exceptions. First, the star selection algorithm previously used was run on brightness maps integrated over the $119-123 \mathrm{~nm}$ region. Due to the loss of sensitivity at $\operatorname{Ly} \alpha$ already recorded prior to JOI, only the calibration in the narrow part of the slit was considered here. A very limited number of stars were found to be valid for calibration at $\operatorname{Ly} \alpha$, making the process challenging to achieve, 

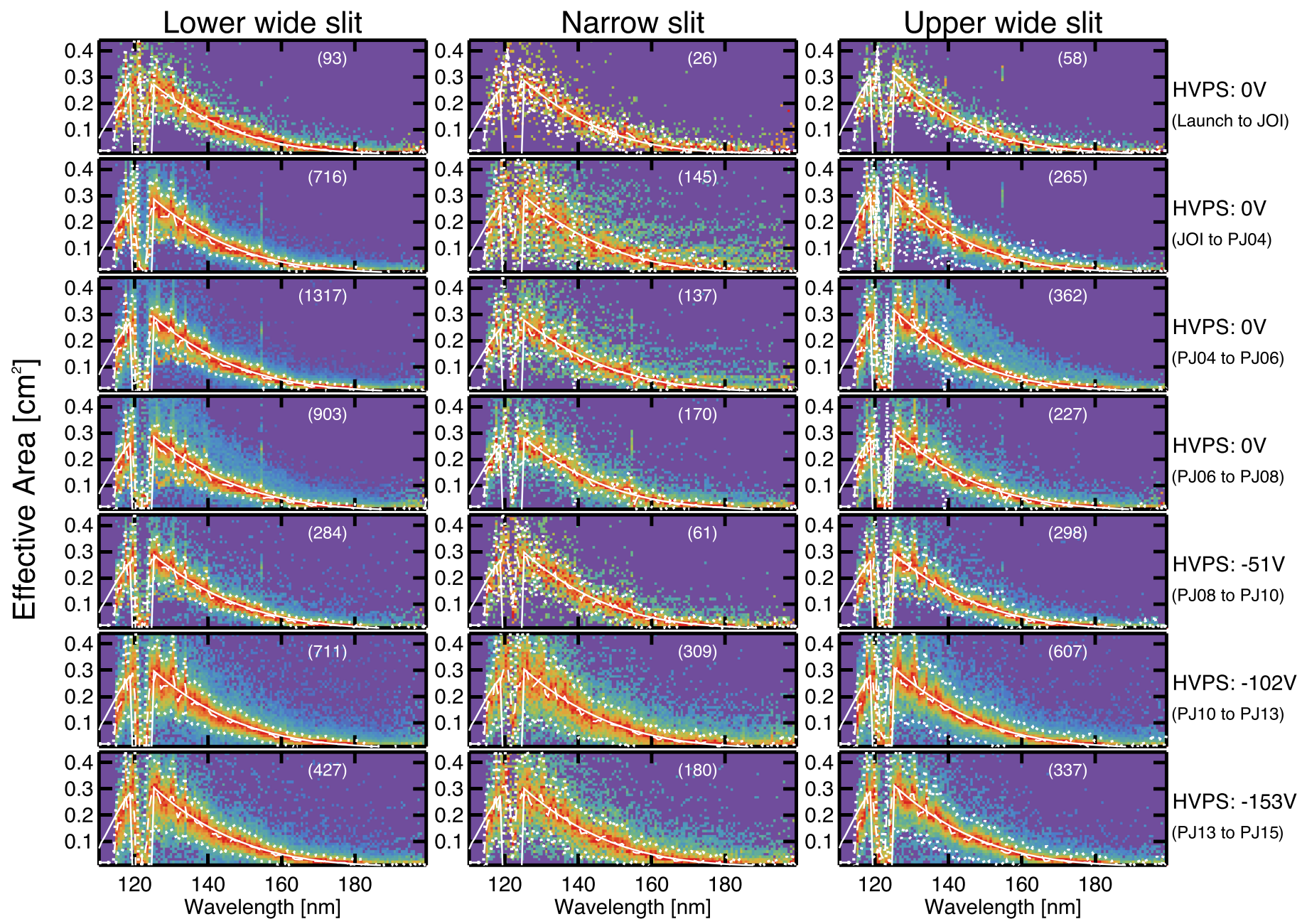

Figure 12. 2D histograms (wavelength vs. effective area) of the measured effective areas for different parts of the slit (left column: lower wide slit; center: narrow slit right column: upper wide slit). The effective area measured over numerous stars at different voltage settings are shown over the different rows. The first row corresponds to the measurements performed during cruise up to JOI at the nominal voltage level. The last row corresponds to the measurements performed between PJ13 and PJ15 at the voltage setting of $-152 \mathrm{~V}$ below the nominal one. Each panel shows the histogram of the measured effective areas for the number of measurements performed at that voltage setting and time, and for that specific part of the slit. The number of measurements used for each panel is shown in the each panel in the upper right corner. The white solid line denotes the averaged effective area obtained after fitting every single effective area measurement. The white dashed line denotes the median effective area from the raw effective measurements. The white dotted lines correspond to the $2 \sigma$ width of the distribution.

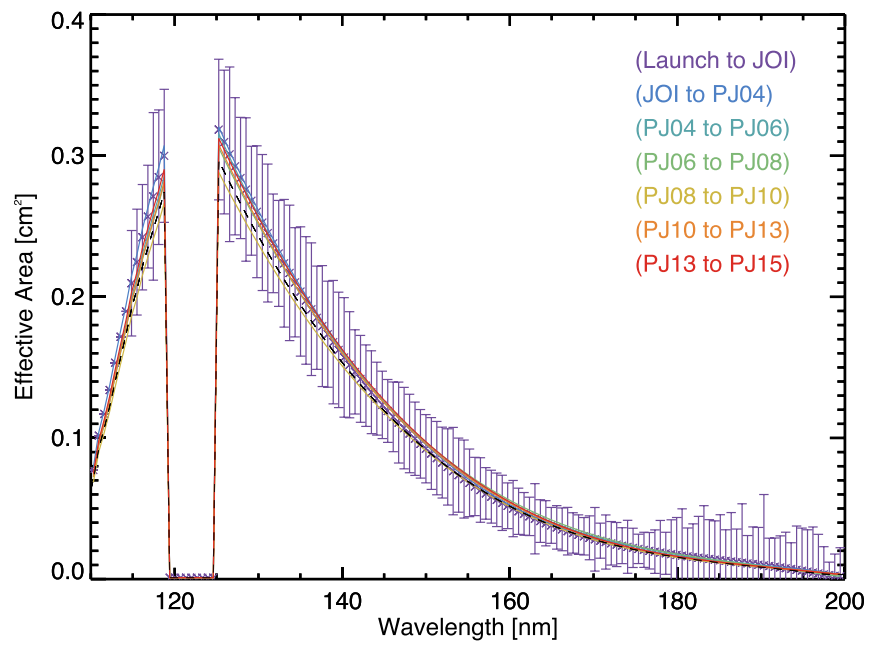

Figure 13. Time series of the upper wide slit effective areas. For clarity, only the uncertainties of the first time span is displayed here (i.e., measurements recorded from launch up to JOI). The black dashed line represents the effective area averaged over from launch to PJ15 at $\mathrm{HV}$ levels ranging from $0 \mathrm{~V}$ above nominal to $+153 \mathrm{~V}$ above nominal.
Table 3

Stars Used for the Ly $\alpha$ Calibration

\begin{tabular}{lccc}
\hline \hline Star Name & $V$-magnitude & Spectral Type & $\begin{array}{c}\text { Number of } \\
\text { Observations }\end{array}$ \\
\hline$\kappa$ Ori (Saiph) & 2.07 & B0.5Ia & 65 \\
$\epsilon$ CMa (Adhara) & 1.50 & B1.5II & 43 \\
$\alpha$ Gru (Alnair) & 1.71 & B6V & 24 \\
$\zeta$ CMa (Furud) & 3.00 & B2.5V & 20 \\
$\alpha$ Pav (Peacock) & 1.92 & B2IV & 12 \\
$\eta$ UMA (Alkaid) & 1.86 & B3V B & 9 \\
$\sigma$ Sgr (Nunki) & 2.07 & B2V B & 2 \\
$\gamma$ Ori (Bellatrix) & 1.64 & B2V C & 2 \\
$\alpha$ Leo (Regulus) & 1.40 & B8IVn & 2 \\
\hline
\end{tabular}

leading to greater uncertainties and a lower level of confidence than for the non-Ly $\alpha$ wavelengths.

Only stars having significant flux at Ly $\alpha$ have been retained to perform the calibration at these wavelengths. Table 3 summarizes the stars used for the Ly $\alpha$ calibration of the narrow slit of UVS. Several of these stars have previously been 


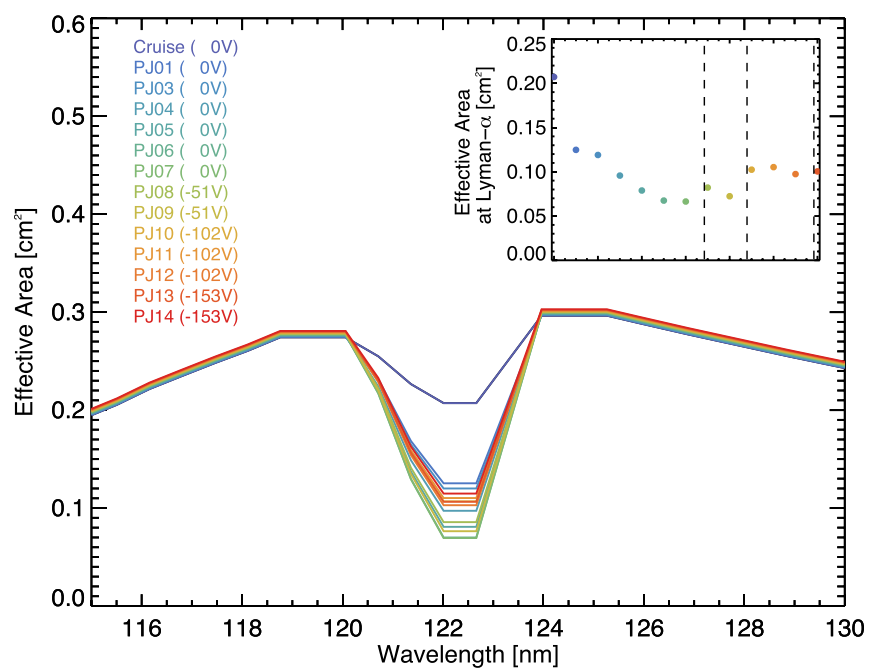

Figure 14. Effective area at Ly $\alpha$ retrieved as a function of PJ. The subplot shows the values of the effective area at $\operatorname{Ly} \alpha$. The potential difference of the voltage of UVS was increased by $-51 \mathrm{~V},-102 \mathrm{~V}$, and $-153 \mathrm{~V}$ below the nominal voltage level prior to PJ8, PJ10, and PJ13, shown by the vertical black dashed lines. Table 3 details the stars used for the calibration. The non-Ly $\alpha$ effective areas, which are identical for each voltage setting, has been slightly shifted up for a better visibility.

classified as variable. The way Juno-UVS takes data, i.e., one limited exposure at each $30 \mathrm{~s}$ spin, and because a large number of spins have to be co-added to build signal, the impact of using few variable stars in the Ly $\alpha$ calibration should be minimal.

The effective area at $\operatorname{Ly} \alpha$ on the narrow slit shows some degradation over time. To investigate this effect, a time-dependent retrieval of the effective area was performed. For each perijove, that quantity was retrieved using all of the stars measured at the voltage setting used during that $\mathrm{PJ}$, and over a period ranging from $+/-1$ PJ ( $\sim 106$ days $)$ around the PJ of interest. Figure 14 shows how this effective area evolved as a function of time. During cruise the measured effective area at Ly $\alpha$ was $0.21 \mathrm{~cm}^{2}$, and had degraded down to $0.07 \mathrm{~cm}^{2}$ by PJ7.

\section{Conclusion/Discussion}

Juno arrived at Jupiter on 2016 July 4 and has been orbiting Jupiter with a 53.5-day period since then. Even though JunoUVS was designed to specifically observe and characterize Jupiter's aurora, a large fraction of the sky has been mapped, since Juno is a spin-stabilized spacecraft. In fact, from launch up to PJ14 (2018 July 16), more than $99 \%$ of the sky has been observed in the $68-210 \mathrm{~nm}$ region. In this work, a subset of stars present in the data were extracted and used to characterize the Juno-UVS detector.

Thanks to the large stellar data set obtained so far, multiple measurements over the same set of stars were used as a way to characterize the instrument response. This provides a powerful way to monitor the health of the instrument, by tracking the PHD evolution over time (see Section 3.3). More specifically, it allows for tracking as the PHD peak approaches the lower end of the event detection discriminator, thus providing a way to assess when the instrument HV level needs to be increased in order to keep a steady response to UV light. Juno-UVS is operating in a harsh radiation environment, especially during PJ observations. During these observations, the fluence on the detector is more than $\sim 40$ times higher than in any other part of the orbit. Even with strategies to preserve the sensitivity of the instrument (see, e.g., Kammer et al. 2018), significant scrubbing of the detector cannot be avoided. The characterization tool developed and presented in this work allows the assessment of when the HV level of the instrument needs to be raised, which was already done prior to PJ8, PJ10, and PJ13.

Second, the calibration of the instrument was performed using a large number of stellar observations described in Section 4. Unlike three-axis stabilized missions, the Juno spacecraft cannot let UVS stare at known stars, as would be good for calibration. Instead, this work presents a more statistical approach. For calibration purposes, the pre-existing UV-spectral databases (IUE, HST/CALSPEC, and HST/ NGSL; see Section 4.1) were refined and a catalog of averaged $I U E+H S T$ stellar UV spectra, degraded to the Juno-UVS resolution, were built in this work. A large number of individual stellar spectra were extracted from the Juno-UVS data (about 8700), between launch and PJ14 (see Section 4.2). Finally, the effective area of the detector was derived from these measurements.

As of $\mathrm{PJ} 14$ and in the non-Ly $\alpha$ region, the instrument response does not show signs of gain loss over time at the nominal HV level, as well at the higher HV levels already used to anticipate for the instrument degradation over time. Prior to arriving at Jupiter, the Ly $\alpha$ sensitivity in the wide parts of the slit already showed signs of significant degradation. In this work, only the calibration at $\mathrm{Ly} \alpha$ in the narrow slit prior to JOI was derived.

We are grateful to NASA and contributing institutions which have made the Juno mission possible. This work was funded by NASA's New Frontiers Program for Juno via contract with the Southwest Research Institute. We thank the entire Juno-UVS engineering and software team for support. The authors want to thank Oswald Siegmund and John Vallerga from Sensor Sciences, LLC, for their contribution and support. The authors thank Andrew Steffl for useful discussions regarding the calibration of ultraviolet spectrograph instruments. This work was supported by JPL contract 1340158 .

\section{ORCID iDs}

Vincent Hue (10) https://orcid.org/0000-0001-9275-0156 G. Randall Gladstone (i) https://orcid.org/0000-0003-0060-072X Thomas K. Greathouse (i) https://orcid.org/0000-00016613-5731

Joshua A. Kammer (1D https://orcid.org/0000-0002-3441-3757 Michael W. Davis (iD https://orcid.org/0000-0003-4338-1635

Bertrand Bonfond (iD https://orcid.org/0000-0002-2514-0187 Maarten H. Versteeg (i) https://orcid.org/0000-0002-25039492

Denis C. Grodent (i) https://orcid.org/0000-0002-9938-4707

Jean-Claude Gérard (i) https://orcid.org/0000-0002-8565-8746

Scott J. Bolton (i) https://orcid.org/0000-0002-9115-0789

Steven M. Levin (i) https://orcid.org/0000-0003-2242-5459

\section{References}

Adriani, A., Filacchione, G., Di Iorio, T., et al. 2017, SSRv, 213, 393 Becker, H. N., Alexander, J. W., Adriani, A., et al. 2017, SSRv, 213, 507 Bohlin, R. C., \& Bianchi, L. 2018, AJ, 155, 162 Bolton, S. J., Adriani, A., Adumitroaie, V., et al. 2017a, Sci, 356, 821 Bolton, S. J., Lunine, J., Stevenson, D., et al. 2017b, SSRv, 213, 5 Bonfond, B., Gladstone, G. R., Grodent, D., et al. 2017, GeoRL, 44, 4463 Broadfoot, A. L., Atreya, S. K., Bertaux, J. L., et al. 1989, Sci, 246, 1459 Connerney, J. E. P., Adriani, A., Allegrini, F., et al. 2017, Sci, 356, 826 
Davis, M. W., Greathouse, T. K., Cooke, C. M., et al. 2016, Proc. SPIE, 9915 , 99152B

Garhart, M. P., Smith, M. A., Turnrose, B. E., Levay, K. L., \& Thompson, R. W. 1997, IUENN, 57, 1

Gladstone, G. R., Persyn, S. C., Eterno, J. S., et al. 2017a, SSRv, 213, 447

Gladstone, G. R., Stern, S. A., Retherford, K. D., et al. 2010, SSRv, 150, 161

Gladstone, G. R., Versteeg, M. H., Greathouse, T. K., et al. 2017b, GeoRL, 44, 7668

Greathouse, T. K., Gladstone, G. R., Davis, M. W., et al. 2013, Proc. SPIE, $8859,88590 \mathrm{~T}$

Grodent, D. 2015, SSRv, 187, 23

Heap, S. R., \& Lindler, D. 2010, BAAS, 42, 494

Jerzykiewicz, M., \& Sterken, C. 1977, AcA, 27, 365

Kammer, J. A., Hue, V., Greathouse, T. K., et al. 2018, Proc. SPIE, 10699, 106993A
Kholopov, P. N. 1982, The New Catalogue of Suspected Variable Stars (Moscow: Nauka)

Kurucz, R. L. 1979, ApJS, 40, 1

Lindler, D., \& Heap, S. 2010, BAAS, 42, 497

Massa, D., \& Fitzpatrick, E. L. 2000, ApJS, 126, 517

Mauk, B. H., Haggerty, D. K., Paranicas, C., et al. 2017, Natur, 549, 66

Nichols, J. S., \& Linsky, J. L. 1996, AJ, 111, 517

Osterman, S., Green, J., Froning, C., et al. 2011, Ap\&SS, 335, 257

Sahnow, D. J., Oliveira, C., Aloisi, A., et al. 2011, Proc. SPIE, 8145, 81450Q

Samus', N. N., Kazarovets, E. V., Durlevich, O. V., Kireeva, N. N., \& Pastukhova, E. N. 2017, ARep, 61, 80

Siegmund, O. H. W. 1999, in ASP Conf. Ser. 164, Ultraviolet-Optical Space Astronomy Beyond HST, ed. J. A. Morse, J. M. Shull, \& A. L. Kinney (San Francisco, CA: ASP), 374

Stern, S. A., Slater, D. C., Scherrer, J., et al. 2007, SSRv, 128, 507

Stern, S. A., Slater, D. C., Scherrer, J., et al. 2008, SSRv, 140, 155 